\title{
Pentylenetetrazol: Posttraining injection facilitates discrimination learning in rhesus monkeys
}

\author{
ROBERT E. BOWMAN and MARK P. HEIRONIMUS \\ University of Wisconsin, Psychology Primate Laboratory, Madison, Wisconsin 53706 \\ and \\ HARRY F. HARLOW \\ University of Arizona, Tucson, Arizona 85721
}

\begin{abstract}
Rhesus monkeys (Macaca mulatta) given immediate posttraining injections of pentylenetetrazol (PTZ) exhibited improved acquisition of difficult discrimination-learning problems (Experiment 2) but not of easy problems (Experiment 1). Optimal facilitation occurred at a dosage of $10 \mathrm{mg} / \mathrm{kg}$. In two further experiments, the interval between training and the injection of $10 \mathrm{mg} / \mathrm{kg}$ of PTZ was varied and optimal facilitation occurred at the 15-min interval. At the 30-min interval, facilitation was absent and error rates were as high as the saline controls, but at the 60-min interval a lower error rate was again seen. All findings were remarkably similar to those previously reported in mice, suggesting a common mechanism for the effect of PTZ on simple discrimination learning in both species. Through the use of the monkey, the potential enhancement of more complex learning by drugs is now accessible to investigation.
\end{abstract}

In 1917, Lashley first demonstrated that pretraining injections of strychnine improved maze-learning performance in rats. This phenomenon was confirmed by McGaugh and Petrinovich (1959), and many subsequent investigations by McGaugh and his colleagues have established the general phenomenon of the enhancement of simple discrimination learning by analeptic (stimulant) drugs in various rodent species (McGaugh, 1966). Since this is the only class of drugs known to improve learning, this phenomenon offers a unique analytical approach to the study of learning and memory. However, the use of pretraining injections raised interpretative difficulties due to possible proactive, nonassociative effects of pretraining injections. Consequently, posttraining injection paradigms using a number of analeptic drugs in subconvulsive doses were introduced by McGaugh (1973). Typically these studies involved dose-ranging studies, i.e., the establishment of dose-effect functions, and/or timeof-injection studies (Krivanek \& McGaugh, 1968; McGaugh \& Krivanek, 1970).

Dose-effect functions are defined as the relationship between dose and "graded effects"' (as measured in individual subjects), whereas dose-response functions are defined as "the proportion of a population that demonstrates a specific effect" at different doses, as proposed in the recent report by Nordberg (1978,

This research was supported in part by Grant MH-11894 from the National Institute of Mental Health and Grant ESO1062 from the National Institutes of Health. p. 38). In prior usage, "dose-response" was often employed to cover both of the definitions cited above; the distinctions reported by Nordberg (1978) are important and will be followed in this paper.

The term "time-of-injection studies" is used here to refer to studies in which the time between termination of training and administration of the drug is varied in order to examine the time-dependent effects of the drug. Previous workers have investigated this parameter, in part to study the nature of hypothetical memory processes.

One critical constraint on the generality of the phenomenon of learning enhancement by stimulant drugs has been the lack of any evidence that this enhancement operates in the primate. The present studies therefore were designed to test this phenomenon in the monkey.

\section{EXPERIMENT 1}

The first experiment was a dose-ranging study intended to establish the dose-effect relationship between pentylenetetrazol (PTZ) and discriminationlearning performance in a group of rhesus monkeys. Doses were chosen on the basis of data from a pilot study in which four monkeys were injected daily with increasing doses of PTZ. At $40 \mathrm{mg} / \mathrm{kg}$, one of the monkeys convulsed. In a subsequent study of the effects of chronic daily injections at fixed dose levels of $30 \mathrm{mg} / \mathrm{kg}$ and lower, the monkey receiving $30 \mathrm{mg} / \mathrm{kg}$ began to show subconvulsive tremoring 
following each daily injection, whereas monkeys at $20 \mathrm{mg} / \mathrm{kg}$ and lower did not. Hence, $20 \mathrm{mg} / \mathrm{kg}$ was selected as the highest dose for the present study, and no overt signs of tremoring or convulsions were ever seen in the studies reported here.

\section{Method}

Four rhesus monkeys (Macaca mulatta), two males and two females, 4-6 years of age, were used. They were all caged individually on light/dark cycles of $16 / 8 \mathrm{~h}$, and were all naive to discrimination learning.

The monkeys were tested in a standard Wisconsin General Test Apparatus, or WGTA (Harlow \& Bromer, 1938), on problems which consisted of pairs of three-dimensional objects selected from the Wisconsin Psychology Primate Laboratory object collection. After adaptation to the WGTA, the monkeys were trained on three conventional WGTA trials per day, each trial consisting of a presentation of the stimulus pair on the test tray. The monkeys were allowed to displace only one of the two objects on a trial (noncorrection procedure) and displacement of the correct object made available a food reward (raisins, marshmallows, or Froot Loops breakfast cereal) in a foodwell concealed by the object. The same pair of stimuli, constituting a problem, were presented daily until the monkey reached a criterion of 9 correct responses in 10 consecutive trials. On the next session after reaching criterion, the monkey was switched to the next condition assigned to it. Errors to criterion were taken as the measure of learning facility in each condition.

The four monkeys were each trained in the WGTA under four different PTZ doses, each dose being associated with the learning of a different problem. The order of the dose-problem conditions was determined by a randomly chosen Greco-Latin square. The PTZ was obtained under the trade name Metrazol from Knoll Pharmaceutical Co. in aqueous solution at physiological $\mathrm{pH}$ in a concentration of $100 \mathrm{mg} / \mathrm{ml}$. The four PTZ doses tested were: zero dose $(.5 \mathrm{ml}$ of physiological saline, constituting the control condition), 5,10 , and $20 \mathrm{mg} / \mathrm{kg}$. The PTZ was injected into the thigh muscle of the restrained, unanesthetized monkey immediately after each daily session in the WGTA.

\section{Results}

The mean numbers of errors to criterion in Experiment 1 for the saline, 5-, 10-, and $20-\mathrm{mg} / \mathrm{kg}$ conditions did not differ significantly from one another. The respective means ( \pm SDs) were $3.75 \pm .75,3.0 \pm$ $1.78,4.0 \pm 1.96$, and $2.75 \pm .85$.

\section{EXPERIMENT 2}

The problems presented in Experiment 1 resulted in such rapid learning and so few errors to criterion that any possible facilitatory effects of the PTZ had too little response space in which to be manifested or to be measured. Consequently, Experiment 2 was conducted, differing from Experiment 1 only in that more difficult discrimination problems were used.

\section{Method}

The same animals, assigned to the same sequences of treatments as in Experiment 1, were again tested in the WGTA, this time using four problems which consisted of pairs of planometric stimulus patterns differing in form but with color and size identical. The two stimuli in each pair will be designated below as Stimulus A and Stimulus B.

\section{Results}

The mean number of errors to criterion in Experiment 2 for the saline, 5-, 10-, and $20-\mathrm{mg} / \mathrm{kg}$ conditions $( \pm$ SEs) were $55 \pm 7.97,39 \pm 16.17,15 \pm 4.61$, and $20 \pm 7.21$, respectively (these numbers are plotted as percentages of the saline value in Figure 1). Three orthogonal contrasts taken within an overall analysis of variance showed that: (1) the saline mean differed significantly from the other three means combined $(\mathrm{F}=9.18, \mathrm{df}=1,6, \mathrm{p}<.05)$, (2) the $5-\mathrm{mg} / \mathrm{kg}$ mean differed significantly from the two higher dose means $[\mathrm{F}=4.22$, df $=1,6, \mathrm{p}<.05$, in this case a unidirectional probability was used based on previous data (Krivanek \& McGaugh, 1968)], and (3) the 10- and $20-\mathrm{mg} / \mathrm{kg}$ means did not differ significantly from one another $(F=.17, \mathrm{df}=1,6, \mathrm{n} . \mathrm{s}$. $)$.

\section{EXPERIMENT 3}

Because of the similarity of the present PTZ doseeffect function to that previously reported in mice (Krivanek \& McGaugh, 1968), a third experiment was undertaken to determine whether a corresponding similarity existed between delay-of-injection functions for the two species.

\section{Method}

Twenty rhesus monkeys (Macaca mulatta), 13 males and 7 females, were used. All were individually caged and all were naive to discrimination learning. Their ages ranged from 2.5 to 3.5 years. The monkeys were tested in the WGTA using a problem which consisted of a pair of planometric stimuli similar to those used in Experiment 2. The animals were randomly divided into five independent groups of four each. Four of the five groups were given PTZ $(10 \mathrm{mg} / \mathrm{kg})$, each at one of the following different delay intervals after training: immediate (Imm), i.e., $<5 \mathrm{~min}$, and 15,30 , and $60 \mathrm{~min}$, while the fifth group was given $.5 \mathrm{cc}$ of saline immediately after testing. Half of the animals in each group were randomly assigned one stimulus object as rewarded, half the other. All other aspects of the procedures were the same as in Experiment 1.

\section{Results}

The respective mean numbers of errors to criterion $( \pm \mathrm{SE})$ for the saline-, imm-, and 15-, 30-, and 60-min groups were $18.75 \pm 6.25,11.25 \pm 2.97,6.75 \pm 1.93$, $19.0 \pm 5.34$, and $11.5 \pm 4.09$. A pronounced objectpreference was seen between the A and B stimuli, as inferred from the fact that the animals assigned object $\mathbf{A}$ as rewarded had consistently lower error rates at all delay intervals than did the animals assigned object B as rewarded. An analysis of variance with four orthogonal contrasts revealed a significant stimulus bias $(\mathrm{F}=10.07, \mathrm{df}=1,10, \mathrm{p}<.01)$, but also indicated that Contrast 1 (the saline and 30-min groups combined vs. the other three groups) was significant $(\mathrm{F}=5.98, \mathrm{df}=1,10, \mathrm{p}<.05)$. This contrast was suggested by previous data in the mouse (Krivanek \& McGaugh, 1968). The other orthogonal 
contrasts, namely Contrast 2 (30-min vs. saline), Contrast 3 (0- and 15-min vs. 60-min), and Contrast 4 (0- vs. 15-min), were all nonsignificant (all Fs $<1$, all $\mathrm{df}=1,10$, n.s.). Contrast 1 exhibited the same profile of mean errors to criterion for the animals trained with object A rewarded as it did for the monkeys trained with object B rewarded despite stimulus bias. This was true both in terms of nearly identical absolute differences between group means at all training-injection intervals and in terms of the nonsignificance of the interaction of stimulus bias with Contrast $1(\mathrm{~F}=.26, \mathrm{df}=1,10, \mathrm{p}>.10)$. In fact, the groups of monkeys trained with object $\mathbf{A}$ rewarded vs. the groups trained with object $B$ rewarded can be viewed as simultaneous, but otherwise independent, replications of the gradient of effect of traininginjection intervals.

\section{EXPERIMENT 4}

In the event that the object preferences shown in Experiment 3 might have biased the results in some unknown fashion (despite the indications to the contrary as discussed above), Experiment 3 was exactly replicated in Experiment 4, with the exception that new problems were used.

\section{Method}

The same subjects and procedures as those in Experiment 3 were used. The new problems each consisted of planometric object pairs which had been equated for preference in a pilot study.

\section{Results}

The respective mean numbers of errors to criterion $( \pm$ SE) for the saline-, Imm-, and 15-, 30-, and 60-min training-injection intervals were $48.5 \pm 8.85,26.75 \pm$ $3.12,25.5 \pm 7.20,48.5 \pm 11.70$, and $33.75 \pm 16.16$. In the analysis of variance done on the data of Experiment 4 , Contrast 1 (saline and 30-min vs. the other three groups) was again significant $(F=4.47, \mathrm{df}=$ $1,15, \mathrm{p}<.05$, based on a unidirectional probability, since the experiment was a replication), while Contrasts 2,3 , and 4 were again all nonsignificant (all Fs $<1$, all df $=1,15$, all ps $>.10$ ). Because of the high similarity across Experiments 3 and 4 in the profiles of error vs. the training-injection intervals, these experiments were averaged together and are shown in Figure 2 as percentages of the average saline condition value.

\section{GENERAL DISCUSSION}

It is well established that learning-task parameters, as well as genetic strain, can strongly influence the outcomes of drug-facilitated learning studies (Krivanek, 1971; Petrinovich, 1967). Because of this, the learning tasks in the present studies were deliberately made as parallel as possible to those given the

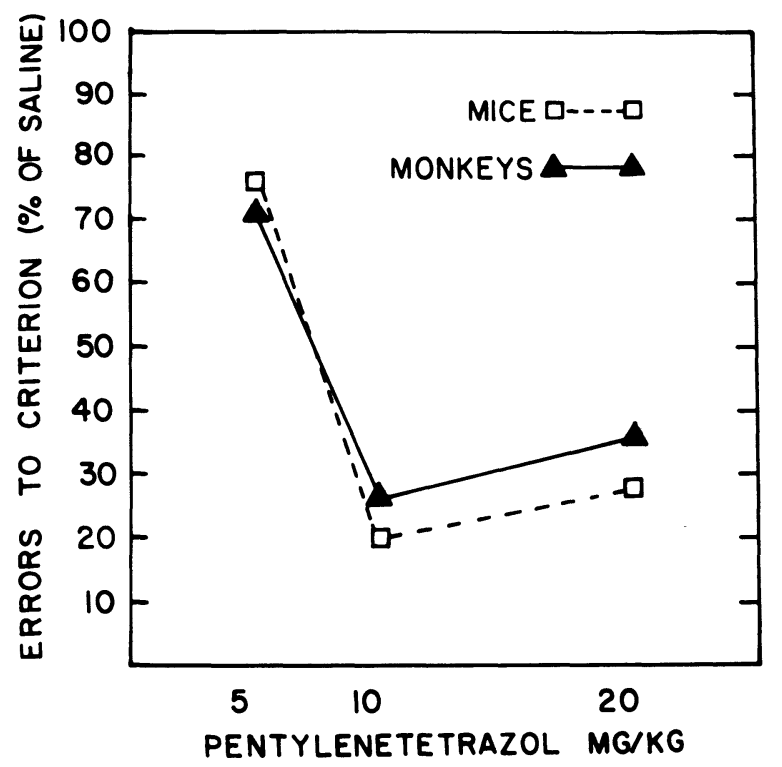

Figure 1. Dose-effect functions of pentylenetetrazol (PTZ) in mice and in monkeys. Errors to criterion are plotted as percentage of saline control group score in each curve. The data for mice are taken from Krivanek and McGaugh (1968).

mice in the Krivanek and McGaugh study (1968), thereby keeping the influence of task variables to a minimum. In both cases, three two-choice visual discrimination trials were run daily (a Y-maze brightness discrimination in the mouse study), with immediate posttraining injections, until a criterion of 9 correct choices in 10 trials was reached. Although Krivanek and McGaugh used more dosage-level conditions and concluded that $15 \mathrm{mg} / \mathrm{kg}$ was the optimum dosage level (and used that dosage in their subsequent delayof-injection study), their $10-$ and $15-\mathrm{mg} / \mathrm{kg}$ conditions did not, in fact, differ significantly. Figure 1 shows that at comparable dosages the functions for the two species line up extraordinarily well.

The species comparison of the PTZ functions for training-injection intervals plotted in Figure 2 (shown as percentages of saline value) also exhibited a strong similarity. The mouse PTZ data were again taken from Krivanek and McGaugh (1968), who used the same discrimination-learning paradigm as in their dose-effect study. The only notable inconsistency between the monkey data and the mouse data, namely the drop in the monkey error rate between Imm and $15 \mathrm{~min}$, is not readily explicable, but it does have a precedent in the rat data of Hunt and Bauer (1969), who found optimal facilitation of a discrimination task using a 15-min delay interval rather than with an immediate injection.

An unexpected feature of the data from both the monkey and the mouse was the reemergence of a facilitatory effect at the 60 -min training-injection interval, after the complete loss of posttraining facilitation at the 30 -min interval. In the mouse, this fea- 


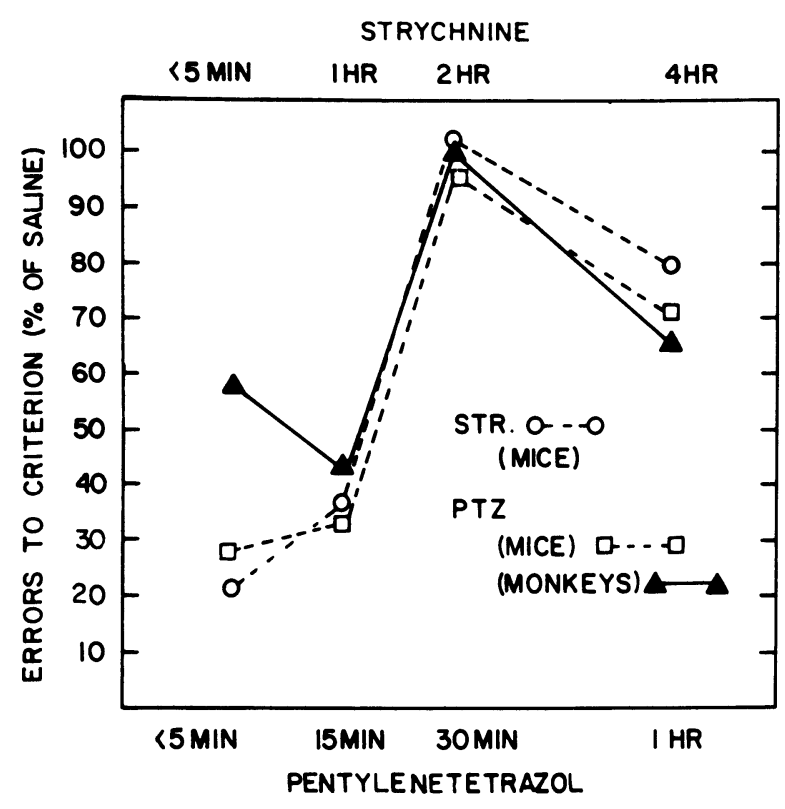

Figure 2. Delay-of-injection functions for mice (Krivanek \& McGaugh, 1968) and monkeys given posttraining injections of pentylenetetrazol (PTZ) and of mice given posttraining injections of strychnine (McGaugh \& Krivanek, 1970). Errors to criterion are plotted as percentage of saline control group score in each curve. Note the expanded delay-of-injection scale (upper horizontal axis) for the STR data.

ture was not peculiar to PTZ, but has also appeared in the learning facilitation induced by strychnine. Figure 2 shows a curve for strychnine effects which is the mean of two essentially identical functions obtained by McGaugh and Krivanek (1970), one at a dosage of $.1 \mathrm{mg} / \mathrm{kg}$ and the other at a dosage of $1.0 \mathrm{mg} / \mathrm{kg}$. Although the training-injection interval as scaled from the mean strychnine curve (upper horizontal axis) has been expanded fourfold for purposes of emphasis, the fact remains that with strychnine, as well as with PTZ, the facilitatory effect of the drug first appeared optimally at short traininginjection intervals, then disappeared at longer traininginjection intervals, and finally reappeared at still longer training-injection intervals with approximately half the facilitatory effect seen at the optimal delay intervals.

The similarity between the delay-of-injection functions for PTZ and strychnine (Figure 2) in the appearance of both an early and a late facilitation limb of the curves, and in the magnitude of the effects seen at both limbs, is highly remarkable, particularly when the fourfold difference in time scale is taken into account. These particular findings should im- pose considerable constraints on any models dealing with the mechanisms underlying the phenomena of drug-facilitated retention.

This demonstration that monkeys exhibit some of the basic phenomena of drug-facilitated learning has major significance for further research in this area. The occurrence of similar dose-effect and traininginjection functions in both mice and monkeys suggests the possibility that the same phenomena may occur in humans. This extrapolation is important because previous work on man has not established these phenomena (Jarvik, 1972) and because further human studies in this area would be difficult, given current ethical concerns. This being the case, the highly developed behavioral repertoire and cognitive capacity of the nonhuman primate recommend these animals as subjects for studies of drug influences on complex learning functions. Such studies represent a potential new line of advance in this area of research.

\section{REFERENCES}

Harlow, H. F., \& Bromer, J. A. A test-apparatus for monkeys. Psychological Records, 1938, 19, 434-436.

Hunt, E. B., \& Bauer, R. H. Facilitation of learning by delayed injections of pentylenetetrazol. Psychopharmacologia (Berlin), 1969, 16, 139-146.

JARVIK, M. E. Effects of chemical and physical treatments on learning and memory. Annual Review of Psychology, 1972, 23, 457-486.

KRIVANEK, J. A. Facilitation of avoidance learning by pentylenetetrazol as a function of task difficulty, deprivation and shock level. Psychopharmacologia (Berlin), 1971, 20, 213-229.

KrivaneK, J. A., \& McGaUgh, J. L. Effects of pentylenetetrazol on memory storage in mice. Psychopharmacologia (Berlin), $1968,12,303-321$.

LASHLEY, K. S. The effects of strychnine and caffeine upon the rate of learning. Psychobiology, 1917, 1, 141-170.

McGaugh, J. L. Time-dependent processes in memory storage. Science, 1966, 153, 1351-1358.

McGaugh, J. L. Drug facilitation of learning and memory. Annual Review of Pharmacology, 1973, 13, 229-241.

McGaugh, J. L., \& KrivaneK, J. A. Strychnine effects on discrimination learning in mice: Effects of dose and time of administration. Physiology \& Behavior, 1970, 5, 1437-1442.

McGaugh, J. L., \& Petrinovich, L. F. The effect of strychnine sulphate on maze-learning. American Journal of Psychology, 1959, 72, 99-102.

NordBe RG, G. F. Factors influencing metabolism and toxicity of metals: A consensus report. Environmental Health Perspectives, 1978, 25, 3-41.

Petrinovich, L. F. Drug facilitation of learning: Strain differences. Psychopharmacologia (Berlin), 1967, 10, 375-378.

(Received for publication March 21, 1979; revision accepted May 18, 1979.) 